Revista Tecné, Episteme y Didaxis: TED. Año 2014, Número Extraordinario. ISSN Impreso: 0121-3814, ISSN web: 2323-0126

Memorias, Sexto Congreso Internacional sobre Formación de Profesores de Ciencias. 08 al 10 de octubre de 2014, Bogotá

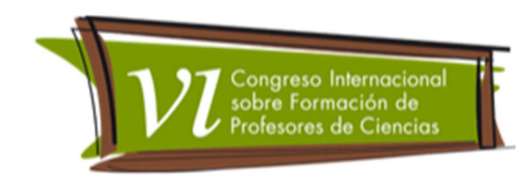

\title{
Relaciones teóricas y prácticas en el currículo de formación de Técnicos en Análisis de Muestras Químicas
}

Alonso Martinez, Maritza Ximena'

Categoría 2. Trabajo de investigación en proceso.

\section{Resumen}

El presente trabajo surge de un proyecto de investigación en desarrollo orientado a indagar la visión que tienen los profesores de las relaciones teórico-prácticas en el currículo de un programa de formación técnica en química articulado con el nivel de educación media.

Para abordar el problema se plantea una metodología de investigación mixta apoyada en el uso de una encuesta tipo Likert que será resuelta por los profesores que orientan este programa a nivel nacional, una entrevista semiestructurada a realizar en unos casos elegidos a conveniencia de la muestra y el análisis documental de las guías académicas planteadas por los profesores para el programa.

En la comunicación se presentan aspectos teóricos, metodológicos y las categorías que orientan la fase inicial de la investigación con el fin de identificar las creencias o pensamientos respecto a cómo se desarrolla la relación teoríapráctica.

\section{Palabras Clave}

Teoría, práctica, currículo, didáctica de las ciencias, enseñanza.

\section{Introducción}

La relación teoría-práctica constituye uno de los principales temas de estudio en el ámbito de la enseñanza de las ciencias, en tal sentido la actividad experimental es uno de los aspectos claves en el proceso de enseñanza y aprendizaje de las ciencias (Wellington, 1998); así mismo el trabajo experimental debe ser considerado como parte de una estrategia que debe ir más allá de la resolución de problemas de lápiz y papel, promoviendo actividades prácticas en

1 Universidad Distrital Francisco José de Caldas. mxalonsom@correo.udistrital.edu.co 
Revista Tecné, Episteme y Didaxis: TED. Año 2014, Número Extraordinario. ISSN Impreso: 0121-3814, ISSN web: 2323-0126 Memorias, Sexto Congreso Internacional sobre Formación de Profesores de Ciencias. 08 al 10 de octubre de 2014, Bogotá

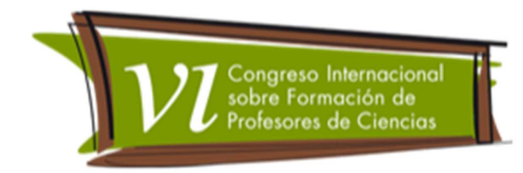

química con el fin de desarrollar temáticas específicas que solamente con la teoría serían difíciles de comprender.

Así mismo Izquierdo y otros (1999) enfatizan en la importancia de la práctica para la formación teórica, considerando que gran parte de los estudiantes no saben aplicar los conocimientos de las ciencias, además «saber y no saber aplicar es no saber». Esta afirmación constituye uno de los principales insumos de la presente investigación, puesto que el programa técnico en Análisis de Muestras Químicas está orientado al ámbito laboral y práctico de la química, en tal sentido, los egresados necesariamente deben demostrar el dominio de las habilidades teórico-prácticas de la ciencia.

De igual manera, un modelo de diseño curricular que establezca las bases para favorecer aprendizajes significativos de un programa técnico en química que a la vez permita relaciones explicitas y coherentes entre aspectos teóricos de la ciencias y aplicaciones prácticas, puede ser orientado al estudiar un Programa Técnico de Análisis de Muestras Químicas con el fin de identificar las relaciones entre aprendizajes teóricos y prácticos; este análisis permite incrementar las oportunidades de aprender de la práctica identificando relaciones entre aspectos teóricos de la ciencias (saber qué) y aplicaciones prácticas (saber cómo) puesto que una problemática común que se evidencia a lo largo de la formación de técnicos es la falta de coherencia entre los contenidos teóricos y la realización de actividades prácticas (tanto de laboratorio como laborales) ya que básicamente se enseña en paradigmas centrados en conocer los conocimientos y no en la construcción de los mismos.

En tal sentido, el presente trabajo surge de una investigación en curso orientada a resolver la siguiente pregunta central investigación: ¿Cómo se relacionan las dimensiones teórica y práctica en la formación de técnicos en análisis de muestras químicas? El desarrollo de este proyecto se justifica en la medida en que sus resultados, a futuro aportarán a la construcción de un currículo innovador para programas técnicos laborales que como se sabe permiten la posibilidad de inclusión de los jóvenes de escasos recursos en el mundo laboral.

\section{Objetivos}

Con el fin de abordar el problema anteriormente enunciado se plantea como objetivo general: Describir las interacciones de las dimensiones teóricas y 
Revista Tecné, Episteme y Didaxis: TED. Año 2014, Número Extraordinario. ISSN Impreso: 0121-3814, ISSN web: 2323-0126 Memorias, Sexto Congreso Internacional sobre Formación de Profesores de Ciencias. 08 al 10 de octubre de 2014, Bogotá

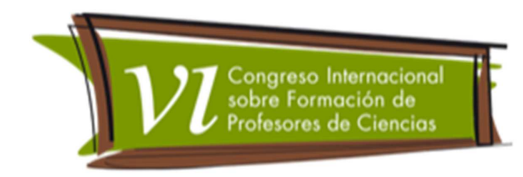

prácticas en al interior de un currículo de formación técnica en química articulado con la educación media.

Los objetivos específicos para desarrollar a lo largo de la investigación son:

- Reconocer las perspectivas acerca de las dimensiones teoría y práctica en el programa de formación técnica en Análisis de Muestras Químicas.

- Identificar la visión de los profesores en torno a la relación teoría-práctica.

- Caracterizar las interacciones teoría-práctica en la formación de técnicos en análisis de muestras químicas.

\section{Marco Teórico}

\section{Currículo}

Para realizar un cambio en el proceso de enseñanza-aprendizaje, según Duschl, "en primer lugar, hemos de reconocer que tendremos que enfrentarnos a muchos casos en los que será necesario modificar el currículo enseñado. Con ello, el problema de determinar cuáles son los contenidos más importantes, no sólo persistirá, sino que será cada vez más acuciante" (1997, p 20). En el contexto de la investigación en educación científica contemporánea, alcanzar evidencias de alfabetización científica implica que los docentes seleccionen contenidos relevantes y justifiquen su importancia y pertinencia en procesos de formación.

Es importante tener en cuenta que el concepto de currículo es muy diverso y por eso se hace necesario definirlo dentro del contexto en el que se esté tratando ya que debido a su visión pueden cambiar los componentes que lo constituyen; cómo plantea Coll: "para situar el curriculum en el contexto de la escolarización, es necesario precisar previamente qué se entiende por curriculum, determinar sus funciones e identificar sus principales elementos, pues el significado y la extensión del término varían enormemente según los autores y las orientaciones teóricas" (1987, pág. 22). De esta manera, el término currículum ha tenido dos acepciones fundamentales: curso de estudios y curso de vida; durante bastante tiempo predominó la primera concepción; sin embargo, recientemente se han producido varios intentos de recuperar el segundo significado, es decir, como conjunto de experiencias vividas en el aula o fuera de ella, pero en relación con la institución escolar.

Clandinin y Connelly (1992, pág. 393) afirman que los profesores no transmiten en las aulas un currículum, sino que viven un currículum y construyen su curriculum, "como un curso de vida". En la misma línea, Pérez (1992, pág. 29) define el 
Revista Tecné, Episteme y Didaxis: TED. Año 2014, Número Extraordinario. ISSN Impreso: 0121-3814, ISSN web: 2323-0126 Memorias, Sexto Congreso Internacional sobre Formación de Profesores de Ciencias. 08 al 10 de octubre de 2014, Bogotá

curriculum como "el relato del conjunto de experiencias vividas por los profesores y alumnos bajo la tutela de la escuela...un proyecto educativo en construcción permanente".

En la teoría educativa el término currículum ha sido utilizado como marco conceptual para entender y determinar la educación y como ámbito y fenómeno de la realidad educativa, así mismo ha dado lugar a planteamientos difusos y ambiguos, determinando de esta manera que como campo de estudio es un concepto sesgado por diversas opciones ideológico-culturales ya que abarca un amplio espectro de la realidad educativa desde diferentes perspectivas.

Gimeno (1988), sugiere una ordenación de definiciones, señalando que el currículum puede analizarse desde cinco perspectivas diferentes:

1. Desde la perspectiva de su funcionalidad social o del enlace entre la sociedad y la escuela.

2. Como proyecto o plan educativo, integrado por diferentes aspectos, experiencias y orientaciones.

3. Como expresión formal y material de ese plan educativo que debe presentar bajo una estructura determinada en sus contenidos y orientaciones.

4. También hay quienes se refieren al currículum como campo práctico.

5. Algunos se refieren a él como un tipo de actividad discursiva, académica e investigadora, sobre los temas propuestos.

Por otro lado, Casarini (1999) afirma que el curriculum puede definirse desde "caminos de aprendizaje" hasta "el instrumento que transforma la enseñanza, guía al profesor y ofrece una retroalimentación y modificaciones al diseño original"; identificando cuatro caminos distintos que las teorías curriculares pueden seguir, teniendo en cuenta; a) suma de exigencias académicas o estructura organizada de conocimiento, b) base de experiencias de aprendizaje, c) sistema tecnológico de producción, d) reconstrucción del conocimiento y propuesta de acción: el puente entre la teoría y la práctica.

En este sentido y para orientar este trabajo, se plantea el currículum como "el proyecto que preside las actividades educativas escolares, precisa sus intenciones y proporciona guías de acción adecuadas y útiles para los profesores que tienen la responsabilidad directa de su ejecución" (Coll, 1987 pág. 31-32). 
Revista Tecné, Episteme y Didaxis: TED. Año 2014, Número Extraordinario. ISSN Impreso: 0121-3814, ISSN web: 2323-0126 Memorias, Sexto Congreso Internacional sobre Formación de Profesores de Ciencias. 08 al 10 de octubre de 2014, Bogotá

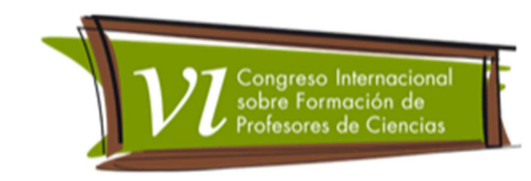

Para Stenhouse (citado por Coll, 1987 pág. 32), el curriculum incluye la descripción del proyecto educativo y el análisis de lo que sucede en el aula cuando el proyecto se desarrolla; desde esta perspectiva existen dos aspectos relacionados con el curriculum que son el proyecto o Diseño Curricular y su aplicación si bien ambos se encuentran íntimamente conectados; así, el curriculum es un eslabón que se sitúa entre la teoría educativa y la práctica pedagógica, entre la planificación y la acción, entre lo que se prescribe y lo que realmente sucede en las aulas. Sin embargo esta amplia definición de curriculum termina por abarcar la totalidad de elementos de la educación formal perdiendo así su carácter específico y también su operatividad, complejizando de esta manera su capacidad de análisis.

En este trabajo y con el fin de subrayar el carácter de proyecto del curriculum se mantendrá la diferencia entre Proyecto o Diseño Curricular y Desarrollo o Aplicación del Curriculum como dos fases de la acción educativa que se nutren mutuamente pero que no se confunden. De esta manera, nuestro propósito es elaborar un diseño curricular que supone, entre otras cosas, traducir principios de índole diversa -ideológicos, pedagógicos, psicopedagógicos- que, tomados en su conjunto, muestran la orientación general del sistema educativo en normas de acción y en prescripciones educativas con el fin de elaborar un instrumento útil y eficaz para la práctica pedagógica (Coll, 1987 pág. 21)

Ahora bien, es propósito de un diseño curricular proceder a un análisis, clasificación, identificación y formulación de las intenciones que presiden el proyecto educativo. Para ello Coll (1987, pág. 50) propone cuatro pasos: elaborar un inventario y selección de las intenciones posibles: ¿qué aspectos del crecimiento personal del alumno trataremos de promover mediante la educación escolar?; en segundo lugar, concretar las intenciones dándoles una formulación que será útiles para guiar y planificar la acción pedagógica; en tercer lugar, plantear el tema de su organización y secuencia de acción temporal y por último, prever una evaluación con el fin de cerciorarse que la acción pedagógica responda a las intenciones perseguidas.

En términos generales este trabajo tendrá en cuenta los siguientes componentes definidores del currículo: las intenciones educativas, la naturaleza de los conocimientos objeto de enseñanza, la secuenciación de estos conocimientos, la metodología de enseñanza y finalmente la evaluación; todo lo anterior visto a la luz de las relaciones teoría-práctica dentro del proceso de formación objeto de estudio. 
Revista Tecné, Episteme y Didaxis: TED. Año 2014, Número Extraordinario. ISSN Impreso: 0121-3814, ISSN web: 2323-0126 Memorias, Sexto Congreso Internacional sobre Formación de Profesores de Ciencias. 08 al 10 de octubre de 2014, Bogotá

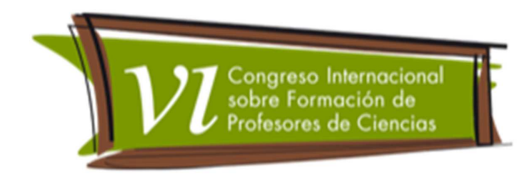

\section{Formación Técnica en Colombia}

Respecto a las intenciones educativas, los procesos de formación han cambiado de acuerdo a las políticas de los gobiernos y las necesidades de las sociedades actuales en un contexto de mercado de trabajo excluyente y precarizado ante los cambios en los perfiles de los trabajadores y las mayores exigencias de requisitos para acceder a ese mercado (que dificultan cada vez más el acceso de los jóvenes). Para Birgin (2008, citado por Ávila et al, 2010) es imprescindible considerar la urgencia por resignificar el papel de los conocimientos, de las instituciones educativas y de la relación entre la educación y el trabajo. Las reformas recientes, alientan una mayor articulación de la educación secundaria, incluida la modalidad académica, general o común, con la formación para el trabajo.

Desde esta perspectiva se hace énfasis en la concepción de la educación media técnica como parte de sistemas más amplios de formación técnico profesional y se tratan las iniciativas de las políticas educativas que promueven múltiples articulaciones: de itinerarios formativos al interior de la modalidad y del nivel medio con la educación superior, con el contexto productivo local y regional, con la educación de jóvenes y adultos y con los requerimientos derivados de las decisiones vocacionales y de las condiciones de vida de los estudiantes.

Para las escuelas, la relación con el mundo del trabajo contribuye a que los estudiantes adquieran conocimientos y competencias en un contexto real, complementando su formación y logrando más herramientas para su inserción laboral posterior (Jacinto y Millenaar, 2007). Dentro de este contexto se encuentra la cultura de una sociedad, entendida como "el conjunto de valores, costumbres, creencias y prácticas que constituyen la forma de vida de un grupo específico" (Eagleton, 2001. pág. 58); ámbito que es necesario analizar ya que permite una aproximación a la realidad de contexto donde finalmente se desempeñarán los jóvenes que se forman en las aulas de clase.

En Colombia, el proyecto "Competencias Laborales, Formación para el Trabajo y Pertinencia de la Educación Media" (Jacinto y Millenaar, 2007, pág. 2) se orienta a facilitar a los estudiantes que cursan los grados $10^{\circ}$ y $11^{\circ}$ en instituciones oficiales y privadas, la oportunidad de adquirir competencias laborales generales y específicas, mediante convenios suscritos entre las instituciones educativas y el sector empresarial. 
Revista Tecné, Episteme y Didaxis: TED. Año 2014, Número Extraordinario. ISSN Impreso: 0121-3814, ISSN web: 2323-0126 Memorias, Sexto Congreso Internacional sobre Formación de Profesores de Ciencias. 08 al 10 de octubre de 2014, Bogotá

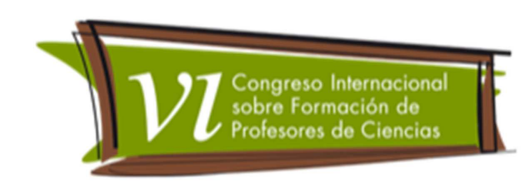

\section{Naturaleza de los conocimientos}

La teoría y la práctica son problemáticas inherentes a todas las actividades humanas, desde la perspectiva filosófica se han definido desde diferentes corrientes del pensamiento que plantean relaciones entre estas fuentes de conocimiento; Chalmers (1997) contempla diferentes corrientes como el falsacionismo en el cual las teorías son construcciones de los seres humanos que buscan dar explicación a los fenómenos del mundo pues éstas "...se construyen como conjeturas o suposiciones especulativas y provisionales que el intelecto humano crea libremente en un intento de solucionar problemas con que tropezaron las teorías anteriores y de proporcionar una explicación adecuada del comportamiento de algunos aspectos del mundo o universo" (pág. 59).

Refiriéndose también al falsacionismo, Chalmers (1997) enfatiza en que "solo se pueden descubrir los secretos de la naturaleza con la ayuda de teorías ingeniosas y perspicaces. Cuanto mayor sea el número de teorías conjeturadas que se enfrentan a la realidad del mundo y cuanto más especulativas sean estas conjeturas, mayores serán las oportunidades de hacer importantes avances en la ciencia" (pág. 67). Es así como desde esta corriente es posible constituir conocimiento científico, en este sentido la ciencia se basa en actualización de teorías.

En otro sentido, para Chalmers (1997) la idea de ciencia desde el empirismo puede ser cuestionada cuando se acude a la generación de teorías basadas en la observación y la experimentación, especialmente en aquellos casos donde "Ios autodenominados "científicos" en esos campos a menudo consideran que siguen en método empírico de la física que para ellos consiste en recopilar "hechos" mediante una observación y una experimentación cuidadosas y en derivar posteriormente leyes y teorías de estos hechos mediante alguna especie de procedimiento lógico" (p. 4), es decir que cuando se asume que algo es ciencia desde la perspectiva fisicalista se presenta una alta correspondencia con los principios empiristas.

Adicionalmente Chalmers advierte que mediante el inductivismo se pueden construir teorías producto de generalizaciones, considerando factores como gran número de repitencia, reproductibilidad y coherencia con las leyes. Esta es la forma de proceder de un inductivista, donde la teoría se ratifica cada vez que una observación no contradice las leyes, es decir que "...ningún enunciado 
Revista Tecné, Episteme y Didaxis: TED. Año 2014, Número Extraordinario. ISSN Impreso: 0121-3814, ISSN web: 2323-0126 Memorias, Sexto Congreso Internacional sobre Formación de Profesores de Ciencias. 08 al 10 de octubre de 2014, Bogotá

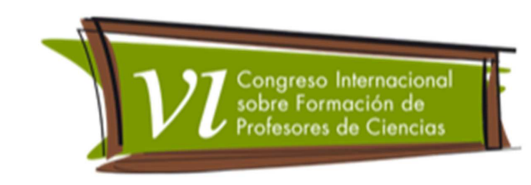

observacional aceptado debe entrar en contradicción con la ley universal derivada" (pág. 15)

Otra corriente filosófica habla sobre el planteamiento de hipótesis y teorías dentro de un cinturón protector que permite falsaciones a través de experimentaciones, donde las teorías son la base de todo el proceso científico, asi mismo dentro del racionalismo los resultados de las comprobaciones experimentales son los que determinan de modo muy sencillo las decisiones de mantener o rechazar una hipótesis.

Kuhn (1962, citado por Porlán, 1989) en su libro la estructura de las revoluciones científicas, plantea el relativismo donde las teorías se generan al interior de un paradigma y son el soporte del mismo y así mismo, a través de la experimentación es posible sustentar los paradigmas; es decir, el paradigma es la guía de la investigación y la interpretación de los fenómenos observables, esto da cabida a la mayor parte de lo que se conoce como la dependencia de la observación por parte de la teoría.

En otro sentido, el objetivismo plantea que las teorías científicas tienen una estructura objetiva en externa a las mentes de los científicos y que los científicos individuales los grupos de científicos pueden o no entender debidamente, así mismo algunas prácticas permiten ampliar el cubrimiento de una teoría es decir que aunque los que plantean la teoría no reconoce sus alcances estas se pueden aplicar para explicar algunos experimentos.

El anterior es un esbozo de la visión de la relación teoría-práctica vista desde las perspectivas tradicionales de la naturaleza del conocimiento.

\section{Secuenciación de los Conocimientos}

De acuerdo a Sanmartí (2000) posterior a la elección de los contenidos se debe plantear cómo secuenciarlos, esta tarea debe obedecer a las finalidades de enseñanza; en el mismo sentido Coll (1987) menciona que la pregunta de ¿cuándo enseñar? se plantea continuamente a la pregunta de ¿qué enseñar? Respecto a la secuenciación Coll (1997) plantea dos grandes vertientes para realizarla, estas son, por análisis de tareas y por análisis de contenidos. Para el análisis de contenidos se plantean un conjunto de técnicas, procedimientos y criterios de secuenciación que se orientan a partir del contenido a enseñar; y en 
Revista Tecné, Episteme y Didaxis: TED. Año 2014, Número Extraordinario. ISSN Impreso: 0121-3814, ISSN web: 2323-0126 Memorias, Sexto Congreso Internacional sobre Formación de Profesores de Ciencias. 08 al 10 de octubre de 2014, Bogotá

el análisis de tareas se contempla un conjunto de técnicas, procedimientos y criterios de secuenciación que parten de los resultados esperados de aprendizaje. Al integrar estos criterios con el objetivo de investigación, es posible decir que el análisis de contenidos se orienta principalmente en la teoría y el análisis de tareas hace énfasis en la práctica.

\section{Enseñanza de las Ciencias}

Al igual que la filosofía, la didáctica de las ciencias ha discutido ampliamente en la relación existente entre la teoría y la práctica, esto debido a la idea generalizada de los profesores al considerar que la ineficacia de la enseñanza se debe a la excesiva transmisión de contenidos conceptuales y al poco desarrollo de trabajos experimentales, lo que conlleva a la búsqueda de un equilibrio entre estos dos tipos de actividades para el aprendizaje de las ciencias (Mosquera, 2011).

Es importante destacar que los enfoques para la enseñanza de las ciencias se basan en los supuestos epistemológicos y la concepción de aprendizaje (Pozo y Gómez, 1998) en tal sentido la teoría y la práctica son subyacentes al enfoque y las metas que este se propone. A continuación se presentan los enfoques más reconocidos para la enseñanza de las ciencias y la visión de la relación teoríapráctica al interior de los mismos.

Dentro de la enseñanza tradicional se hace énfasis en la teoría donde los conocimientos suelen presentarse como saberes acabados y establecidos que son trasladados a los estudiantes bajo una visión estática y absoluta del saber científico. Las clases se basan en exposiciones del profesor acompañadas con algunos ejercicios o demostraciones que sirven para ilustrar las explicaciones.

En otro sentido se plantea la corriente de enseñanza por descubrimiento donde el énfasis es en la práctica, basando la enseñanza en experiencias que permitan a los estudiantes investigar y reconstruir los principales descubrimientos científicos. Desde este enfoque los productos de la ciencia son una consecuencia directa del dialogo entre el método y la naturaleza.

Desde la perspectiva de la enseñanza expositiva se enfatiza nuevamente en la teoría y la idea es lograr que los estudiantes asuman como propios los significados científicos, esto a través de un acercamiento progresivo de las ideas de los 
Revista Tecné, Episteme y Didaxis: TED. Año 2014, Número Extraordinario. ISSN Impreso: 0121-3814, ISSN web: 2323-0126 Memorias, Sexto Congreso Internacional sobre Formación de Profesores de Ciencias. 08 al 10 de octubre de 2014, Bogotá

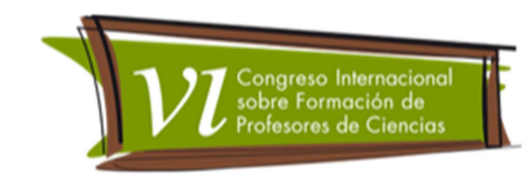

estudiantes a los conceptos científicos haciendo necesaria la explicitación de las relaciones entre los conocimientos previos y la nueva información.

Al interior del modelo de enseñanza mediante el conflicto cognitivo se parte de las concepciones alternativas para confrontarlas con situaciones conflictivas con el fin de lograr un cambio conceptual; desde esta perspectiva el énfasis es nuevamente en la teoría entendiendo el logro del conocimiento como la sustitución de las concepciones iniciales por otras teorías más próximas al conocimiento científico.

En la enseñanza mediante investigación dirigida se fomenta el aprendizaje autorregulado, donde no se espera que el estudiante descubra por sí mismo los conocimientos científicos, sino que la selección y sucesión de problemas le oriente para que aprenda, a partir de fuentes diversas, los contenidos que se estiman relevantes en una disciplina dada. Dado que el alumno debe movilizar constantemente sus conocimientos y que existe una interrelación continúa entre teoría y aplicación práctica, el aprendizaje basado en problemas puede conseguir una mejor integración de los conocimientos declarativos y procedimentales.

El modelo de enseñanza por explicación y contrastación de modelos se plantea una integración de la teoría y la práctica, donde el papel de la enseñanza se fundamenta en la explicación de modelos alternativos, creando diversos escenarios explicativos para hacer dialogar a los diversos modelos e interpretaciones posibles de los fenómenos estudiados contrastándolos entre sí y reescribiendo unos en otros.

Finalmente en una sociedad, donde los conocimientos cambian con una velocidad impresionante, se hace necesario que los ciudadanos aprendan flexible y eficazmente, que puedan articular los conocimientos que elaboran entre el saber qué y el saber cómo, y para ello deben adoptar y poner en escena unos procedimientos que les permitan efectivamente resolver problemas y aplicar los nuevos conocimientos aprendidos en diversidad de situaciones. De esta manera, es preciso aprender no solo contenidos conceptuales, sino también aprender a predisponerse ante el mundo y ante la sociedad y aprender a desarrollar capacidades científicas, lo cual implica el aprendizaje de contenidos actitudinales y de contenidos procedimentales. Así mismo es necesario considerar explícitamente la integración entre teoría y práctica como aspectos indisolubles en la enseñanza de las ciencias (Mosquera, 2011). 
Revista Tecné, Episteme y Didaxis: TED. Año 2014, Número Extraordinario. ISSN Impreso: 0121-3814, ISSN web: 2323-0126 Memorias, Sexto Congreso Internacional sobre Formación de Profesores de Ciencias. 08 al 10 de octubre de 2014, Bogotá

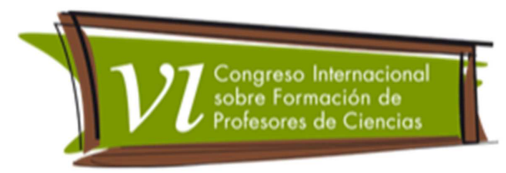

\section{Metodología}

Este trabajo se plantea desde un enfoque mixto de investigación con un tipo de estudio descriptivo a través de modelos de intervención de análisis documental con el propósito de analizar el diseño curricular de un Programa de Formación en Química articulado con la Educación Media Técnica.

Para lograr los objetivos propuestos en este trabajo esta propuesta prevé implementarse en el estudio de un programa de formación técnica en Química que actualmente se encuentra en ejecución en articulación con la Educación Media Técnica en el Servicio Nacional de Aprendizaje SENA a nivel nacional; el propósito es evidenciar la visión de los profesores respecto a la relación teoríapráctica del actual currículo de formación en química con el fin de comprender las dinámicas de la formación.

Se analizó información sobre el campo de la relación teoría-práctica vista desde perspectivas epistemológicas y didácticas; con el fin de plantear categorías (Ver tabla 1) de análisis basadas en las diferentes corrientes epistemológicas para adquisición del conocimiento tales como la inductivista, falsacionista, racionalista, relativista, objetivista e instrumentalista; así mismo se analiza la relación teoría-práctica desde diferentes enfoques de enseñanza como lo son el tradicional, enseñanza por descubrimiento, modelo expositivo, enseñanza mediante conflicto cognitivo, la investigación dirigida, y la contrastación de modelos.

Tabla 1. Categorías de Análisis

\begin{tabular}{|c|c|c|}
\hline CATEGORIA & $\begin{array}{c}\text { INDICADORES DE } \\
\text { ANÁLISIS }\end{array}$ & DESCRIPCIÓN \\
\hline \multirow{2}{*}{ 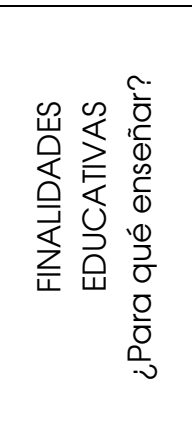 } & $\begin{array}{l}\text { CONTEXTO DE } \\
\text { FORMACIÓN }\end{array}$ & $\begin{array}{l}\text { Ideas de los profesores en relación con a quienes está } \\
\text { dirigido el programa de formación, teniendo en cuenta } \\
\text { edades, conocimientos previos y contexto socio-cultural. }\end{array}$ \\
\hline & PERFILES DE EGRESO & $\begin{array}{l}\text { Conocimiento de los objetivos de formación. } \\
\text { Visión de la pertinencia entre la formación y el campo } \\
\text { laboral - impacto social -impacto académico (pertinencia } \\
\text { de los conocimientos para aplicarlos en el campo del } \\
\text { análisis de muestras químicas) } \\
\text { Percepción de la pertinencia de los conocimientos en } \\
\text { relación con expectativas profesionales a futuro. }\end{array}$ \\
\hline
\end{tabular}


Revista Tecné, Episteme y Didaxis: TED. Año 2014, Número Extraordinario. ISSN Impreso: 0121-3814, ISSN web: 2323-0126 Memorias, Sexto Congreso Internacional sobre Formación de Profesores de Ciencias. 08 al 10 de octubre de 2014, Bogotá

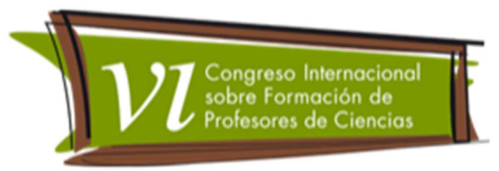

\begin{tabular}{|c|c|c|}
\hline & $\begin{array}{l}\text { COMPETENCIAS } \\
\text { ESPERADAS }\end{array}$ & $\begin{array}{l}\text { Ideas acerca de las competencias a desarrollar en los } \\
\text { estudiantes de acuerdo con el Proyecto Educativo del SENA } \\
\text { y con propósitos educativos constructivistas. } \\
\text { Los profesores articulan competencias conceptuales y } \\
\text { competencias prácticas en los estudiantes }\end{array}$ \\
\hline & OBJETIVOS & $\begin{array}{l}\text { Ideas de los profesores en relación con la manera cómo se } \\
\text { articulan los objetivos del PFT con los objetivos de desarrollo } \\
\text { personal, la socialización, la productividad económica y el } \\
\text { aprendizaje general. }\end{array}$ \\
\hline \multirow{7}{*}{ 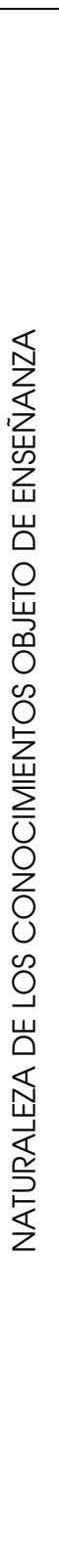 } & $\begin{array}{c}\text { POSTURA } \\
\text { INDUCTIVISTA }\end{array}$ & $\begin{array}{l}\text { Las teorías se construyen a partir del análisis de } \\
\text { observaciones hechas a diferentes fenómenos. La fuente de } \\
\text { la verdad no es la lógica, sino la experiencia. Ningún } \\
\text { enunciado observacional aceptado debe entrar en } \\
\text { contradicción con la ley universal derivada. }\end{array}$ \\
\hline & $\begin{array}{c}\text { POSTURA } \\
\text { FALSACIONISTA }\end{array}$ & $\begin{array}{l}\text { La ciencia progresa mediante el ensayo y el error, debido a } \\
\text { que la situación lógica hace imposible la derivación de } \\
\text { leyes y teorías universales a partir de enunciados } \\
\text { observacionales. Es posible validar una teoría desde su } \\
\text { resistencia a la falsación. }\end{array}$ \\
\hline & $\begin{array}{c}\text { POSTURA } \\
\text { RACIONALISTA }\end{array}$ & $\begin{array}{l}\text { Las teorías son la base de todo el proceso científico. La } \\
\text { observación depende de la teoría. Sólo se puede llevar a } \\
\text { cabo una experimentación precisa si se tiene una teoría } \\
\text { susceptible de proporcionar predicciones en la forma de } \\
\text { enunciados observacionales precisos. }\end{array}$ \\
\hline & POSTURA RELATIVISTA & $\begin{array}{l}\text { Las teorías se generan al interior de un paradigma y son el } \\
\text { soporte del mismo. A través de la experimentación es } \\
\text { posible sustentar los paradigmas; estos guían la } \\
\text { investigación y la interpretación de los fenómenos } \\
\text { observables, dando cabida a la mayor parte de lo que se } \\
\text { conoce como la dependencia de la observación por parte } \\
\text { de la teoría. }\end{array}$ \\
\hline & POSTURA OBJETIVISTA & $\begin{array}{l}\text { Teorías científicas tienen una estructura objetiva y externa a } \\
\text { las mentes de los científicos. Algunas prácticas permiten } \\
\text { ampliar el cubrimiento de una teoría, es decir que aunque } \\
\text { quienes trabajan en ella no reconocen todos sus alcances, } \\
\text { esta puede aplicarse para explicar algunos experimentos. }\end{array}$ \\
\hline & $\begin{array}{c}\text { POSTURA } \\
\text { INSTRUMENTALISTA }\end{array}$ & $\begin{array}{l}\text { La finalidad de la ciencia es producir teorías que definen } \\
\text { mecanismos e instrumentos desde los cuales se interpretan } \\
\text { situaciones observables. }\end{array}$ \\
\hline & POSTURA EMPIRISTA & $\begin{array}{l}\text { La experiencia es fuente del conocimiento científico. El } \\
\text { método científico se concibe como un conjunto de reglas } \\
\text { de aplicabilidad universal, para observar fenómenos e inferir } \\
\text { conclusiones a partir de ellos. La observación es objetiva y } \\
\text { no depende de las ideas u otras características de la } \\
\text { persona que observa. }\end{array}$ \\
\hline
\end{tabular}


Revista Tecné, Episteme y Didaxis: TED. Año 2014, Número Extraordinario. ISSN Impreso: 0121-3814, ISSN web: 2323-0126

Memorias, Sexto Congreso Internacional sobre Formación de Profesores de Ciencias. 08 al 10 de octubre de 2014, Bogotá

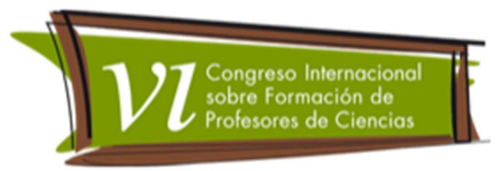

\begin{tabular}{|c|c|c|}
\hline \multirow{3}{*}{ 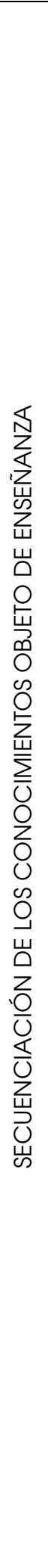 } & $\begin{array}{l}\text { ÉNFASIS EN TAREAS } \\
\text { (PRÁCTICA) }\end{array}$ & $\begin{array}{l}\text { Se plantean teniendo en cuenta una experimentación } \\
\text { cuidadosa a partir de los resultados, de acuerdo a los } \\
\text { siguientes pasos: 1. Análisis de las actividades objeto de } \\
\text { enseñanza, 2. Secuenciación de actividades siguiendo la } \\
\text { estructura lógica de la acción, 3. Análisis de la eficacia de } \\
\text { la secuencia, y finalmente 4. Revisión de la secuencia según } \\
\text { los resultados de la experimentación. } \\
\text { En esta lógica se tiene en cuenta la tarea, la secuencia y el } \\
\text { resultado; no se tiene en cuenta el funcionamiento } \\
\text { cognitivo. } \\
\text { Se inspira en programas instruccionales como ejecución } \\
\text { eficaz de tareas; en tal sentido el énfasis es meramente } \\
\text { pragmático. }\end{array}$ \\
\hline & $\begin{array}{l}\text { ÉNFASIS EN } \\
\text { CONTENIDOS } \\
\text { (TEORÍA) }\end{array}$ & $\begin{array}{l}\text { Se prescribe una secuencia descendente donde las } \\
\text { secuencias de aprendizaje tienen que ordenarse partiendo } \\
\text { de los conceptos más generales y avanzando de forma } \\
\text { progresiva hacia los conceptos más específicos, pasando } \\
\text { por los conceptos intermedios. } \\
\text { Esta postura se centra de forma prioritaria y exclusiva en los } \\
\text { componentes conceptuales del contenido, de tal manera } \\
\text { que el único tipo de criterio útil para establecer secuencias } \\
\text { es el concerniente a las relaciones entre conceptos. }\end{array}$ \\
\hline & $\begin{array}{l}\text { ENLACE DE } \\
\text { CONCEPTOS, } \\
\text { PRINCIPIOS Y } \\
\text { PROCEDIMIENTOS }\end{array}$ & $\begin{array}{l}\text { Se presenta en un principio una panorámica global de las } \\
\text { principales partes del contenido de la enseñanza, pasando } \\
\text { seguidamente a elaborar cada una de ellas y regresando } \\
\text { periódicamente a la visión de conjunto con el fin de } \\
\text { enriquecerla y ampliarla. } \\
\text { Sobre una misma materia se puede aspirar a que el } \\
\text { aprendizaje de los alumnos concierna fundamentalmente a } \\
\text { los aspectos conceptuales, a los aspectos teóricos o a los } \\
\text { aspectos de procedimiento. Sin embargo sea cual sea el } \\
\text { contenido organizador elegido, los otros dos están siempre } \\
\text { en mayor o menor grado como contenidos de soporte. } \\
\text { Dependiendo del aspecto que se utilice como origen, se } \\
\text { tiene: } \\
\text { 1. Primacía de la orientación conceptual: utiliza una } \\
\text { estructura conceptual para su organización. La base } \\
\text { incluye los conceptos más fundamentales y } \\
\text { representativos de esta estructura, que son también los } \\
\text { más generales e inclusivos. } \\
\text { 2. Primacía de la orientación procedimental: utiliza una } \\
\text { estructura de procedimientos para su organización. La } \\
\text { base incluye el procedimiento o procedimientos más } \\
\text { simples y al mismo tiempo más generales; es decir los } \\
\text { que implican un menor número de pasos o de acciones } \\
\text { y se aplican a una gama más amplia de situaciones. } \\
\text { 3. Primacía de la orientación en los principios: utiliza una }\end{array}$ \\
\hline
\end{tabular}


Revista Tecné, Episteme y Didaxis: TED. Año 2014, Número Extraordinario. ISSN Impreso: 0121-3814, ISSN web: 2323-0126 Memorias, Sexto Congreso Internacional sobre Formación de Profesores de Ciencias. 08 al 10 de octubre de 2014, Bogotá

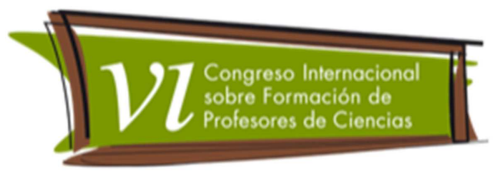

\begin{tabular}{|c|c|c|}
\hline \multirow{8}{*}{ 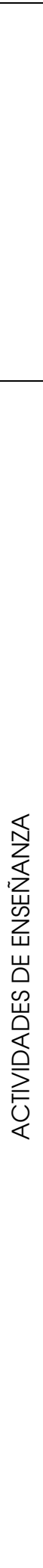 } & & $\begin{array}{l}\text { estructura teórica para su organización. La base incluye } \\
\text { los principios más simples y fundamentales. }\end{array}$ \\
\hline & HISTÓRICO & $\begin{array}{l}\text { Los contenidos del curso se abordan teniendo en cuenta su } \\
\text { desarrollo cronológico lo que implica la valoración de los } \\
\text { cambios fuertes o débiles que ocurren al interior de las } \\
\text { teorías (las relaciones teoría y práctica están imbricadas y } \\
\text { se interpretan a la luz del momento histórico que se estudia). }\end{array}$ \\
\hline & $\begin{array}{l}\text { INTEGRACIÓN } \\
\text { PROBLEMICA }\end{array}$ & $\begin{array}{l}\text { Los contenidos se abordan como necesidad de ampliar los } \\
\text { marcos explicativos de los estudiantes sobre situaciones } \\
\text { problema. }\end{array}$ \\
\hline & $\begin{array}{l}\text { ENSEÑANZA } \\
\text { TRADICIONAL }\end{array}$ & $\begin{array}{l}\text { El énfasis de este modelo son los conceptos; la mente de los } \\
\text { alumnos debe seguir la lógica del discurso científico y por lo } \\
\text { tanto la meta es llenarla de saberes conceptuales. Se sigue } \\
\text { una ruta lógica marcada por los saberes disciplinares. Se } \\
\text { basa en la transmisión de saberes conceptuales. }\end{array}$ \\
\hline & $\begin{array}{l}\text { ENSEÑANZA POR } \\
\text { DESCUBRIMIENTO }\end{array}$ & $\begin{array}{l}\text { Su énfasis es la práctica. Se fomenta a toda costa la } \\
\text { actividad autónoma de los alumnos. De acuerdo con este } \\
\text { enfoque, la enseñanza se basa en el planteamiento y } \\
\text { resolución de situaciones abiertas en las que el alumno } \\
\text { pueda reconstruir los principios y leyes científicas siguiendo } \\
\text { los pasos que los científicos han usado en su momento. } \\
\text { Con su énfasis en la observación y en la formulación de } \\
\text { hipótesis, este enfoque tiene mucho que ver con } \\
\text { concepciones excesivamente inductivistas sobre la ciencia } \\
\text { y el trabajo científico. }\end{array}$ \\
\hline & $\begin{array}{l}\text { ENSEÑANZA } \\
\text { EXPOSITIVA }\end{array}$ & $\begin{array}{l}\text { Su énfasis es la teoría. La idea es lograr que los alumnos } \\
\text { asuman como propios los significados científicos, a través } \\
\text { de un acercamiento progresivo de sus ideas a las teorías y } \\
\text { los conceptos científicos. } \\
\text { En este modelo, los conocimientos actitudinales y } \\
\text { procedimentales quedan relegados a un segundo plano. }\end{array}$ \\
\hline & $\begin{array}{l}\text { ENSEÑANZA } \\
\text { MEDIANTE EL } \\
\text { CONFLICTO } \\
\text { COGNITIVO }\end{array}$ & $\begin{array}{l}\text { El énfasis es la teoría y los conceptos. Se parte de las } \\
\text { concepciones alternativas de los estudiantes para ser } \\
\text { confrontadas en situaciones conflictivas con el fin de lograr } \\
\text { un cambio conceptual, entendido como la sustitución de } \\
\text { un concepto o teoría por otros más potentes, es decir más } \\
\text { próximos a los acuñados por el conocimiento científico. } \\
\text { Los núcleos conceptuales de la ciencia constituyen el eje } \\
\text { del currículo y los contenidos procedimentales y } \\
\text { actitudinales no desempeñan apenas ningún papel en la } \\
\text { organización del currículo. }\end{array}$ \\
\hline & $\begin{array}{l}\text { ENSEÑANZA } \\
\text { MEDIANTE } \\
\text { INVESTIGACIÓN } \\
\text { DIRIGIDA }\end{array}$ & $\begin{array}{l}\text { Este modelo integra la teoría y la práctica. La investigación } \\
\text { de los estudiantes se constituye en un laborioso proceso de } \\
\text { construcción social de teorías y modelos, apoyado no } \\
\text { solamente en ciertos recursos metodológicos sino también } \\
\text { en el despliegue de actitudes. }\end{array}$ \\
\hline
\end{tabular}


Revista Tecné, Episteme y Didaxis: TED. Año 2014, Número Extraordinario. ISSN Impreso: 0121-3814, ISSN web: 2323-0126 Memorias, Sexto Congreso Internacional sobre Formación de Profesores de Ciencias. 08 al 10 de octubre de 2014, Bogotá

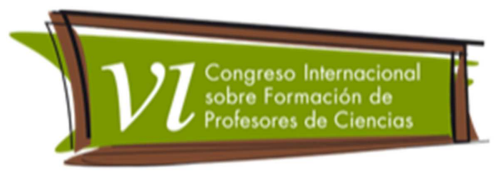

\begin{tabular}{|c|c|c|}
\hline & & $\begin{array}{l}\text { Se fomenta el aprendizaje autorregulado, donde no se } \\
\text { espera que el estudiante descubra por sí mismo los } \\
\text { conocimientos científicos, sino que la selección y sucesión } \\
\text { de problemas le oriente para que aprenda, a partir de } \\
\text { fuentes diversas, los contenidos que se estiman relevantes } \\
\text { en una disciplina dada. } \\
\text { Dado que el alumno debe movilizar constantemente sus } \\
\text { conocimientos y que existe una interrelación continúa entre } \\
\text { teoría y aplicación práctica, el aprendizaje basado en } \\
\text { problemas puede conseguir una mejor integración de los } \\
\text { conocimientos declarativos y procedimentales. }\end{array}$ \\
\hline & $\begin{array}{l}\text { ENSEÑANZA POR } \\
\text { EXPLICACIÓN Y } \\
\text { CONTRASTACIÓN DE } \\
\text { MODELOS }\end{array}$ & $\begin{array}{l}\text { El núcleo organizador son los modelos, es decir la forma en } \\
\text { que se representa el conocimiento existente en un dominio } \\
\text { dado. El papel de la enseñanza se fundamenta en la } \\
\text { explicación de modelos alternativos, creando diversos } \\
\text { escenarios explicativos para hacer dialogar a los diversos } \\
\text { modelos e interpretaciones posibles de los fenómenos } \\
\text { estudiados contrastándolos entre sí y redescribiendo unos } \\
\text { en otros. }\end{array}$ \\
\hline \multirow{6}{*}{ 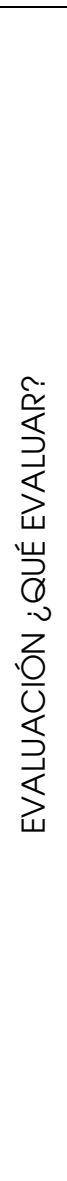 } & TRADICIONAL & $\begin{array}{l}\text { Los alumnos deben devolver al profesor el conocimiento de } \\
\text { la forma más precisa (reproductiva) posible. Se utilizan } \\
\text { ejercicios repetitivos comprobando el grado de dominio de } \\
\text { la rutina o sistema de resolución previamente explicado. }\end{array}$ \\
\hline & $\begin{array}{l}\text { POR } \\
\text { DESCUBRIMIENTO }\end{array}$ & $\begin{array}{l}\text { Se tiene en cuenta la forma en que se llega al } \\
\text { conocimiento conceptual; para tal efecto se utilizan guías } \\
\text { que replican experimentos cruciales que se han seguido en } \\
\text { el pasado y que han permitido el desarrollo del nuevo } \\
\text { conocimiento. }\end{array}$ \\
\hline & EXPOSITIVA & $\begin{array}{l}\text { La evaluación se centra casi que exclusivamente en la } \\
\text { manera como los estudiantes justifican y describen } \\
\text { conocimientos conceptuales así como su capacidad para } \\
\text { relacionar unos conceptos con otros. Se emplean con } \\
\text { frecuencia mapas conceptuales y diagramas heurísticos. }\end{array}$ \\
\hline & $\begin{array}{l}\text { CONFLICTO } \\
\text { COGNITIVO }\end{array}$ & $\begin{array}{l}\text { Se busca que los estudiantes compartan los conocimientos } \\
\text { científicos y abandonen sus ideas iniciales. Se emplean } \\
\text { mapas conceptuales, diagramas heurísticos, informes, } \\
\text { carteles, etc. }\end{array}$ \\
\hline & $\begin{array}{l}\text { INVESTIGACIÓN } \\
\text { DIRIGIDA }\end{array}$ & $\begin{array}{l}\text { Las actividades de enseñanza se conciben en sí mismas } \\
\text { como actividades de evaluación, las cuales están } \\
\text { orientadas a la resolución de problemas. Se emplean } \\
\text { mapas conceptuales, diagramas heurísticos, informes, } \\
\text { carteles, etc. }\end{array}$ \\
\hline & $\begin{array}{l}\text { CONTRASTACIÓN DE } \\
\text { MODELOS }\end{array}$ & $\begin{array}{l}\text { Enriquecimiento de los modelos elaborados por los propios } \\
\text { alumnos a partir de las discusiones con sus compañeros, las } \\
\text { explicaciones del profesor y las evaluaciones recibidas. }\end{array}$ \\
\hline
\end{tabular}


Revista Tecné, Episteme y Didaxis: TED. Año 2014, Número Extraordinario. ISSN Impreso: 0121-3814, ISSN web: 2323-0126 Memorias, Sexto Congreso Internacional sobre Formación de Profesores de Ciencias. 08 al 10 de octubre de 2014, Bogotá

La encuesta tipo Likert y las categorías que la orientan se encuentran en proceso de validación por expertos y posterior a este, se aplicará a los profesores del Sena que a nivel nacional orientan el programa de formación técnica en química (45 en total); esto se realizará a través de una plataforma virtual y se plantean afirmaciones en las cuales los profesores deberán seleccionar una de cuatro opciones (Ca: completamente de acuerdo, A: de acuerdo, D: en desacuerdo, y Cd: completamente en desacuerdo.

Con base en análisis de estos datos se planteará una entrevista semiestructurada a 5 docentes elegidos convenientemente dentro de la población con el fin de determinar bajo qué perspectiva se está desarrollando el actual proceso de formación y con base en ello reconocer sus fortalezas y debilidades respecto al aprendizaje de las ciencias y la consideración de contenidos conceptuales, procedimentales y actitudinales.

Además de la entrevista se espera poder realizar un análisis documental del programa de formación (documento proporcionado por el Sena) y de las guías académicas que plantean los docentes para el desarrollo de las competencias todo esto a la luz de las categorías anteriormente mencionadas.

\section{Resultados esperados}

A través de esta investigación se busca contar con la participación de todos los docentes que a nivel nacional orientan el programa con el fin de tener una visión global de la problemática planteada; así mismo, como producto de esta investigación se busca caracterizar las relaciones teórico-prácticas inherentes al proceso de formación de técnicos en análisis de muestras químicas, vistas desde las perspectivas de los docentes del programa.

\section{Conclusiones}

El diseño curricular orienta la próctica pedagógica y de esta manera debe ser fuente de reflexión constante ya que de la misma dependen los avances en el aprendizaje que puedan tener los estudiantes.

La relación teoría-práctica en la enseñanza de las ciencias debería corresponder a los intereses por resolver un problema relacionando aspectos tanto teóricos como experimentales en la misma dinámica. 
Revista Tecné, Episteme y Didaxis: TED. Año 2014, Número Extraordinario. ISSN Impreso: 0121-3814, ISSN web: 2323-0126 Memorias, Sexto Congreso Internacional sobre Formación de Profesores de Ciencias. 08 al 10 de octubre de 2014, Bogotá

Esta investigación se plantea como medio para construir criterios de análisis de las dimensiones académicas de la relación teoría-práctica al interior del programa de formación técnica en análisis de muestras química implementado en Instituciones Educativas del nivel medio con el apoyo del SENA. Con el propósito de garantizar criterios de calidad para la investigación, se tienen en cuenta las posturas planteadas por Ruiz (2003), donde se ofrecen pasos o metodologías para acertar con los criterios de validez de la investigación cualitativa; en este sentido las preguntas y categorías se someterán a validación por expertos, garantizando así la credibilidad dada por técnicas de triangulación, la dependencia determinada por la consistencia de los datos recogidos, y la consistencia interna del proyecto.

Como resultado de esta investigación se espera constituir una propuesta curricular del programa de formación técnica profesional en química para el Servicio Nacional de Aprendizaje SENA en el marco de articulación con la educación media que contribuya a mejorar el aprendizaje teórico-práctico.

\section{Referencias bibliográficas}

Ávila, Lucrecia; Caciorgna, Laura; Caballero, Luciana; Guinsburg Kiper, Natalia. (2010) Material para planificar la enseñanza de la formación para el trabajo. Córdoba.

Casarini, M. (1999). Teoría y diseño curricular.(2 @ edición). Monterrey, México. Trillas-UV.

Chalmers, Alan F. (1997). ¿Qué es esa cosa llamada ciencia? Una valoración de la naturaleza y el estatuto de la ciencia y sus métodos. Décimo novena edición. Siglo XXI Editores. España.

Clandinin, D. J. Y Connelly, F. M. (1992). Handbook of research on curriculum. New York: McMillan.

Coll, Cesar (1987) Psicología y Currículum. Una aproximación psicopedagógica a la elaboración del currículum escolar. España: Cuadernos de Pedagogía. Editorial Paidos.

Duschl, Richard A. (1997) Renovar la Enseñanza de las Ciencias. Importancia de las teorías y su desarrollo. Narcea, S.A. de Ediciones. Madrid. 
Revista Tecné, Episteme y Didaxis: TED. Año 2014, Número Extraordinario. ISSN Impreso: 0121-3814, ISSN web: 2323-0126

Memorias, Sexto Congreso Internacional sobre Formación de Profesores de Ciencias. 08 al 10 de octubre de 2014, Bogotá

Eagleton, Terry. (2001). La idea de cultura. Una mirada política sobre los conflictos culturales. España: Editorial Paidos.

Gimeno S., J. (1988). El currículum: una reflexión sobre la práctica. Madrid: Morata.

Ibañez, N; Castillo, R. (2011) Lineamientos estratégicos para la formación de competencias laborales Negotium, vol. 6, núm. 18, enero-abril, 2011, pág. 88101. Fundación Miguel Unamuno y Jugo. Maracaibo: Venezuela.

Izquierdo, Mercè; Sanmartí, Neus y Espinet, Mariona (1999). "Fundamentación y diseño de las prácticas escolares de ciencias experimentales". Revista Investigación didáctica, Vol.17 (1), pág. 45-59. Barcelona: Universitat Autónoma de Barcelona.

Jacinto, Claudia y Millenaar, Verónica (2007). Las relaciones entre escuelas y empresas: un camino con nuevos desafíos en América Latina. Tendencias en foco. Artículo publicado en Boletín redEtis no7, diciembre de 2007, pág. 1 a 6.

Mosquera Suárez, Carlos Javier (2011) Perspectivas contemporáneas de la investigación en didáctica de las ciencias experimentales. Memorias Congreso de Investigación y Pedagogía II Nacional y I Internacional. Perspectivas, retos y transformaciones en contextos educativos. Tunja.

Pérez G., A. (1992). ¿Qué son los contenidos de la enseñanza?". En J. Gimeno y A. Pérez (Coords.): Comprender y transformar la enseñanza. Madrid: Morata.

Ruiz, J.I (2003) Técnicas de Triangulación y Control de Calidad en la Investigación Socioeducativa. España: Ediciones Mensajero

Sanmartí, Neus (2000). Diseño de unidades didácticas. En Perales, F. y Cañal P. (Coords.), Didáctica de las Ciencias experimentales. Alcoy: Editorial Marfil

Wellington, J. (1998). Practical work in science. En Wellington (Coord.), Practical work in school science. Which way now? (pág. 3-16) Londres: Routledge. 